\title{
Genetic and Environmental Factors in Health-related Behaviors: Studies on Finnish Twins and Twin Families
}

\author{
Jaakko Kaprio', Lea Pulkkinen ${ }^{2}$ and Richard J. Rose ${ }^{3}$ \\ 'University of Helsinki and National Public Health Institute, Helsinki, Finland \\ ${ }^{2}$ University of Jyväskylä, Jyväskylä, Finland \\ ${ }^{3}$ Indiana University, Bloomington, Indiana and University of Helsinki, Finland
}

\begin{abstract}
Family, twin and adoption studies have provided evidence for Familial and genetic influences on individual differences in disease risk and in human behavior. Attempts to identify individual genes accounting for these differences have not been outstandingly successful to date, and at best, known genes account for only a fraction of the familiality of most traits or diseases. More detailed knowledge of the dynamics of gene action and of specific environmental conditions are needed. Twin and twin-family studies with multiple measurements of risk factors and morbidity over time can permit a much more detailed assessment of the developmental dynamics of disease risk and the unfolding of behavioral risk factors.
\end{abstract}

In Finland, two longitudinal studies of adolescent twins and their families, each with about 2700 participating families, form a complementary, ongoing study base: FinnTwin 16, initiated in 1991 (twins born 1974-1979) and FinnTwin12, initiated in 1994 (twins born 1983-1987). The fourth and third waves, respectively, of data collection are now in progress. Because of the young age of this data base, the primary focus at present is on behavioral risk factors, but that focus will evolve to disease outcomes on later follow-up.

These cohorts will be used a) to analyze gene by environment interaction in well-characterized subsets of the cohort, assessing behavioral risk factors such as smoking, alcohol use and physical activity, and b) to examine genetic and environmental determinants of change and stability of behavioral risk factors and disease risk using multivariate, quantitative genetic models. Genetic and environmental effects vary over the life-span and across different environmental conditions. Only longitudinal studies in genetically informative data sets permit the study of such effects.

After a decade of research with the older Finnish twin cohort (Kaprio, 1994), it was recognized in the mid-1980s that development of younger twin cohorts for prospective studies would be of great utility. While studies of adult pairs are of great use for examining the role of genetic and environmental factors in many chronic diseases, we became interested in the development of behaviors that are relevant for maintaining health or increase the risk of illness. Studies of children, adolescents and young adults are needed for such studies. Accordingly, linkages were made to Finland's
Central Population Registry (CPR) to include twins born through 1987 to the earlier cohort which had included twins born through 1957.

\section{Datasets}

In 1986, a new twin panel was established from all twin pairs (including parents of twins) born in Finland during 1958-1986. It consists of 21,958 twin pairs (estimated 6114 monozygotic, 7922 same-sexed dizygotic and 7922 pairs of opposite sex) (Kaprio et al., 1990). Multiple births were identified through the use of family member links existing for all persons in the CPR. Thus persons born on the same day to the same mother were considered multiples. From 1974 onwards, it corresponds closely to the number of twin births recorded by Statistics Finland. In August 1998, twins born in 1987 ( $N=597$ pairs) were added to the database for the FinnTwin 12 study (see below).

\section{FinnTwin16 Study}

One major project based on the younger twin cohort is the FinnTwin 16 Study, which is a longitudinal study of five consecutive birth cohorts (1975-1979) of Finnish twins, their siblings and parents, initiated in 1991 and funded by the National Institutes of Health, USA, and headed by Professor Richard Rose of Indiana University. After baseline data-collection was completed in January 1996, pairwise response rates exceed $88 \%$, yielding baseline data on 2733 twin pairs. Parents and older sibs have returned questionnaires at similar response rates, yielding data on substantial numbers of extended twin families.

The baseline assessment of all twin pairs, made within 60 days of their 16th birthdays, includes a survey of health habits and attitudes, a symptom checklist, MMPI personality scales, and relationships with parents, peers, and co-twin. All respondent twins were surveyed again at age 17 , again on a monthly basis, with the measures of health habits and health-relevant attitudes, symptom reports, and social relationships. From 1993 onwards, the third survey

Address for correspondence: Jaakko Kaprio, Professor of Genetic Epidemiology, Dept. of Public Health, University of Helsinki, PO Box 41FIN-00014 Helsinki, Finland. Email:jaakko.kaprio@helsinki.fi 
has been carried out based on four annual mailings, two in the autumn, two in spring, timed to ensure that respondents were on average aged 18.5 years. The response rate was $95 \%$ and this data collection was completed by August, 1998. Starting in the autumn of 2000, we have semi-annually contacted the annual birth cohorts, with contact so far with those born 1975-1977. The response rates for these cohorts exceed 88\%. During 2002, twins born in 1978 and 1979 are being contacted again to complete the fourth wave of data collection.

The goal of the study is to carry out analyses of genetic and environmental contributions to consistency and change in health-related behaviors during late adolescence and early adulthood. These are all periods of significant change in adolescents' and young adults' health-habits and in their relationships to peers and parents. In addition, the representative sampling of male, female, and opposite-sex twins in this study are used to evaluate gender modulation of longitudinal effects and gender modulation of the genetic and environmental relationships among health habits, attitudes, and personality.

From the FinnTwin 16 database, we have invited twin pairs concordant and discordant for their alcohol use developmental trajectory for further study. Starting in the spring of 2001, we are studying pairs using a structured psychiatric interview, blood tests, neuropsychological testing, and EEG/ERP measures. We aim to study some 250-300 pairs in this NIH-funded study. A small substudy is targeting $\mathrm{MZ}$ pairs concordant and discordant for obesity and eating disorders identified on the basis of the fourth survey for detailed metabolic and imaging studies. The clinical phase started in January 2002.

\section{FinnTwin12 Study}

Another five birth cohort study, the FinnTwin12 Study, was started in September 1994 to examine genetic and environmental determinants of precursors of health-related behaviors, with particular focus on use and abuse of alcohol, in initially 11 - to 12 -year-old twins. Major funding was obtained from the National Institutes of Health, USA. This research is cast within the perspective of developmental genetic epidemiology, asking whether precursors of risk behaviors are evident to parents, teachers, and classroom peers as early as age 12 .

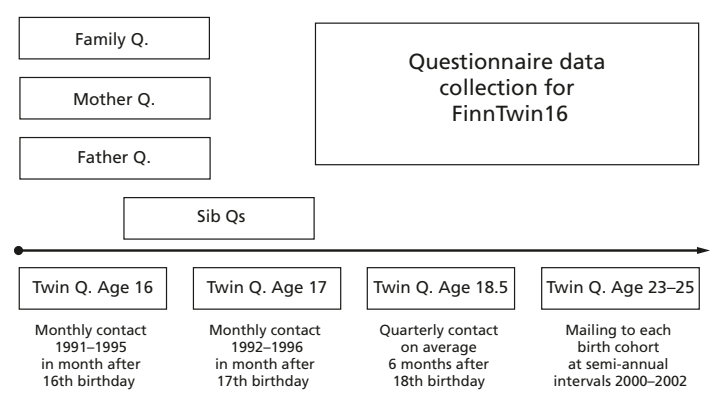

Figure 1

Flow chart of data collection procedures in the FinnTwin16 study.
The study has a two-stage sampling design. The larger, first-stage study is an epidemiological investigation of five consecutive and complete birth cohorts of Finnish twin children, including questionnaire assessments of both twins and parents at baseline, starting with a family questionnaire (returned by 2724 families, $87 \%$ participation rate) that was mailed late in the year before the twins reach age 12, with follow-up of all twins at age 14 and, in recently initiated data collection, again at age 17.5 years. For the epidemiological study of Stage-1, we excluded families in which one or both co-twins were deceased or living outside Finland, families in which both co-twins lived apart from both biological parents, and families in which the CPR contained no residential address for a twin, implying that that twin was in an institutional care facility and would be unable to participate with the demands of our study.

Five separate questionnaires were mailed at baseline to each twin family: A family questionnaire, usually completed by the twins mothers, which when returned, was immediately followed by a postal mailing of individual questionnaires to both parents and the two co-twins. Parents not resident with their twins were also contacted. The family questionnaire requested basic information on the twins' gestation and delivery and early development, their zygosity, and the specific school in which they were then enrolled. The baseline questionnaire assessment of the twins' parents includes an 11-item diagnostic screen for alcohol-related problems, enabling us to identify twin children at elevated risk of substance abuse. The baseline questionnaire assessment of parents, and their twins, are complete for all five birth cohorts. The twins' questionnaire included items on self-reported height and weight; time spent with twin and friends, time spent watching TV, videos, playing computer games, playing other indoor games, listening to music, playing an instrument, reading, painting, doing handcrafts, hanging around with friends indoors/outdoors, going to clubs, exercising; 22 items of relationship with parents, atmosphere and caring at home; life satisfaction and pubertal development. At age 12, several months after the baseline questionnaires had been returned, we also sought a rating from parents and classroom teachers of all twins in the epidemiological sample, using a Multidimensional Peer Nomination Inventory (MPNI; Pulkkinen et al., 1999) developed for this research by Lea Pulkkinen. Ratings were completed by $93 \%$ of teachers, and $92 \%$ of parents of the entire Stage-1 twin sample.

At age 14, most of the items asked of the twins at age 11-12 were repeated. In addition, items on frequency of use of alcohol and intoxication, use by peers of alcohol, own smoking status and peer smoking, alcohol expectancies, self-esteem, and peer use of drugs were included. The age 14 follow-up assessment of all twins, by postal questionnaire, is complete for the first four birth cohorts and virtually complete for the remaining one (twins born in 1987); current participation rate to date is $88 \%$.

A second follow-up questionnaire, at age 17 and a half years, was initiated in autumn of 2000 , with over $90 \%$ participation for the 1983 cohort contacted between autumn 1983 and spring of 2001 . The 1984 cohort is currently being contacted. Again, many items are repeated, with 


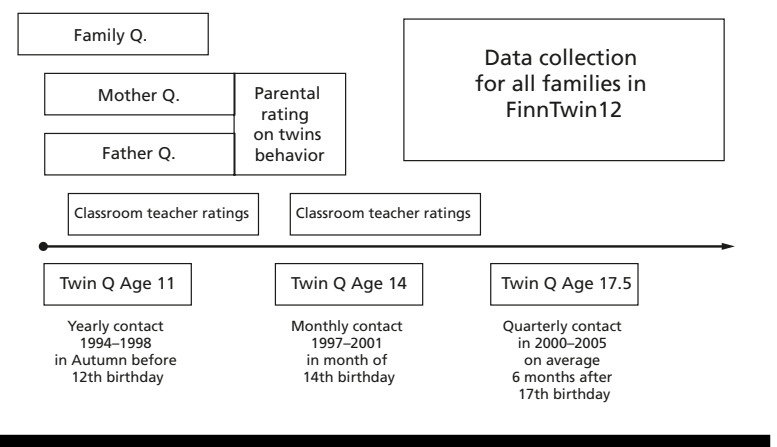

Figure 2

Flow chart of data collection procedures for all twin families in the FinnTwin12 study

additional age-specific items on health behaviors. We also ask for mobile phone and Internet use, as new means of social interaction that rapidly have become widespread among youth in Finland.

This epidemiological first-stage of our Finn Twin 12 study thus includes some 5600 twins, and 5,000 of their biological parents, and, as expected from population-based ascertainment, the twins form equal proportions of brother-brother, sister-sister, and brother-sister pairs, permitting robust testing of gender modulation of genetic and environmental risks in the development of health behaviors, and appraisal of the magnitude and persistence of effects of variation in pubertal timing.

Nested within this epidemiological, population-based study, is the second-stage of FinnTwin 12, an intensive assessment of a sub-sample of twin families. Most of the sub-sample is selected at random, but this random sample is then enriched with twins at elevated familial risk for alcoholism. For inclusion in the intensively-studied sub-sample of Stage-2, we further required that (one or both of) the twins' parents returned the family questionnaire and gave us written permission to initiate school contact, that the family questionnaire contained no new information about the twins (e.g., studying abroad, living at home but severely handicapped) that made them ineligible for inclusion in the study; we then further required that both twins and at least one parent be Finnish-speaking, because it was cost-prohibitive to train and conduct structured psychiatric interviews in Swedish, as well as Finnish, language. About 6\% of the Finnish population speak Swedish as their mother tongue - all questionnaires were made in both languages. We have carefully assessed the non-responders at each stage, and found no evidence for selection for family type (both vs. single parents), parental age, area of residence, type or sex of twin.

Of the families for whom permission was obtained for school contact, 1035 were selected for intensive (Stage-2) study. These included 135 families from the 1983 cohort, 202 from 1984, 206 from 1985, and 207 from 1986, and 285 from 1987. About half are families in which the twins are assumed to be at elevated risk for alcohol problems, given the elevated scores on the Malmö-modified Michigan Alcoholism Screening Test (Mm-MAST) (Seppä et al., 1990), self-reported by one or both of their biological parents at baseline. We created an 11-item lifetime version of the Mm-MAST, adding two additional items to increase the predictive validity of the Mm-MAST as a screen for DSM-III-R/IV diagnoses of alcohol abuse and dependency. We have also found that the Mm-MAST is an effective screen for alcohol problems assessed by interview. Interviews of the parents in these families, using the SemiStructured Assessment for Genetics of Alcoholism (SSAGA) instrument (Bucholz et al., 1994) have been completed, with a total of 1864 interviews completed (participation rate was approximately 90\%).

In-school assessments of the twins, including peer nominations and individual behavioral testing, have been completed for all five cohorts. In Finnish culture, twin children are usually placed in the same classrooms; in this study, both co-twins were in the same class for nearly $90 \%$ of the twin pairs. The twins of the present study were in 1002 classes, with a mean class size of 25 students. The twins had 23,200 classmates of the same age; 11,297 girls and 11,903 boys, for whom peer nominations were also obtained. These data provide an extraordinary resource to compare the different aspects of the behaviors of twins and singletons in an exceptionally large and representative study population.

The adolescent SSAGA interviews are complete for the first four cohorts, yielding 1333 completed interviews $(88.7 \%)$. The interviews of the last cohort will be completed by early 2002. The twins' interviews (with C-SSAGA-A, which provides diagnoses and symptom counts of several psychiatric disorders, including alcohol abuse and dependence, drug abuse and dependence, conduct disorder, oppositional disorder, depression, anxiety, suicidal behavior, anorexia, and bulimia) are highly standardized for the adolescent twins' age, given the very dynamic nature of alcohol use and risk-associated behaviors in mid-adolescence; accordingly, we aim to complete $90 \%$ of adolescent interviews within six months of the 14 th birthday. We found no systematic source of bias in the small subset of parents and twins whom we have not been able to interview. Adolescent interviews are conducted faceto-face using custom software and a notebook PC to enter the twin's responses online. In addition neuropsychological tests (Trails, Mazes, California Stroop) are conducted, and

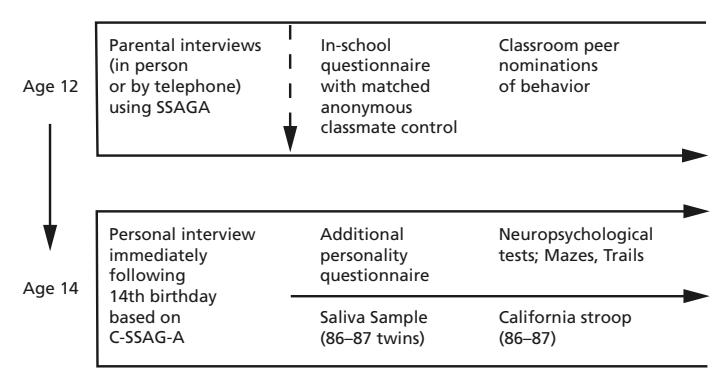

Figure 3

Additional assessments done for "intensively" studied subsample of FinnTwin12 families. 
for the 1986 and 1987 saliva samples for hormonal assays (cortisol, testosterone) have been collected.

The FinnTwin 12 dataset represents a very rich longitudinal design, with a genetically informative component (twins and parents), with assessments of twins' behavior based on self, co-twin, peer, parental and teacher reports. We plan continuing assessments of both the total epidemiological sample, as well as the more intensively studied subsample as the twins age.

\section{Analyses of Existing Data to Date}

There are many analyses in progress based on these cohorts and the data that has been collected. In the following we briefly, and not exhaustively, describe some reports from the main areas of interest.

\section{Alcohol Use in Adolescence}

As an example of a recently completed analysis, we have examined predictors of initiation of alcohol use at age 14 (Rose et al., 2001). First, pairwise analyses and genetic modeling indicated that common environmental (i.e., familial non-genetic) effects were equivalent in male and female adolescents and accounted for $76 \%$ of the total variation in abstinence/drinking. Secondly, logistic regression analyses, among 2206 individuals (twins) with complete data on risk-relevant measures at both baseline and followup, identified significant predictors of drinking/abstaining at age 14 , including female gender, twin sibling of opposite-sex, accelerated pubertal development, and the twins' assessments, made at age 12, of reduced parental monitoring and less supportive home atmosphere; drinking at age 14 was predicted also by behaviors rated by the twins' classroom teachers two years earlier, increasing with rated behavioral problems, but decreasing with rated emotional problems. Thus, environmental factors shared by twin siblings account for most of the variance in abstaining or drinking at age 14, and we could identify predictors of drinking in the adolescent twins' home environments, and in their dispositional behaviors, sibling interactions, and pubertal timing (Rose et al., 2001).

Among twins at age 16 years, we have shown that familial effects on alcohol use and abstinence are contextdependent. They vary according to the region of residence in Finland: families residing in communities with a high average alcohol consumption having a smaller influence on their children's drinking patterns than families from communities with a low average consumption (Rose et al., 1999). Furthermore these contextual factors also affect longitudinal changes in alcohol use parameters (Rose et al., 2001). Danielle Dick, as part of her doctoral thesis, has shown that characteristics of communities, such as the proportion of young adults, between community migration rates, and local alcohol sales all moderate the genetic influences on alcohol use in adolescence (Dick et al., 2001).

Some, but not all of the differences between communities in abstinence rates in adolescence can be accounted for by differences in language and religiosity (Winter et al., in press). Religiosity itself has somewhat different shared and unshared components of variance when examined by region and sex (Winter et al., 1999).

\section{Weight, Height and Obesity}

Kirsi Pietiläinen is a medical doctoral student, who is studying longitudinal determinants of obesity and body size from birth onwards in twin-families from the FinnTwin 16 study (Pietiläinen et al., 1999; Pietiläinen et al., 2001).These studies provide population-based estimates of the contribution of genetic factors to interindividual variability in liability to obesity, which is a necessary parameter for all studies seeking to identify individual susceptibility genes. We have earlier shown that there is a strong genetic correlation between BMI and pubertal development (Kaprio et al., 1995). These data are also informative about the development of gene action on body size through development. Karri Silventoinen, Ph.D., studied sex differences in height using the Finntwin 16 families, and did not find evidence for sex-specific gene action (Silventoinen et al., 2001).

\section{Allergy and Asthma}

The doctoral dissertation by Dr. Maija Räsänen reported the cumulative incidence of asthma and hay fever among two generations of Finns. As she has summarized in her dissertation, the genetic contribution to variance in liability to both asthma and hay fever was found to be high (Laitinen et al., 1998; Räsänen et al., 1998). In addition to corresponding parental disease, a high ponderal index at birth and maternal smoking also proved to be risk factors for asthma (Räsänen et al., 2000), while male gender, small sibship and gestational maturity proved to be independent risk factors for hay fever (Räsänen et al., 1997). The relative contribution of genes vs. environment in disease liability and the effect of perinatal risk factors for asthma and hay fever varied according to parental disease status.

\section{Physical Activity}

Also, we are investigating the tracking of physical activity and factors predicting changes in physical activity habits during late adolescence among young twins based on the FinnTwin 16 data set. Marja Aarnio, Master in Health Administration, is doing her doctoral thesis on this project. Her first two papers explored the relationship of leisure physical activity in adolescents with other health habits and social factors (Aarnio et al., 1997), and the familial aggregation in three generations of physical activity (Aarnio et al., 1997). Her last two articles examine the correlates of persistence in physical activity and inactivity over a 30-month period from age 16 to age 18.5 years.

\section{Personality}

Analyses of the contribution of genes and environment to personality traits at different ages are central in our studies, and have utilized the multimethod, multirater design in the FinnTwin 12 study. Elina Vierikko, a psychology doctoral student in Jyväskylä, has examined sex differences and rater differences in aggressive behaviors from the baseline study of Finntwin12 (Vierikko et al., 2002). The results suggested significant genetic, common environmental, and specific environmental effects on aggression in both boys and girls, but the best fitting model differed depending on whether the teacher or parent was the informant. For both ratings, boys showed lower heritability and higher levels of common environmental effects than girls. In addition, the 
teacher rating data also suggested the presence of either sexspecific common environmental effects or sex-specific genetic effects.

In a study of depressive symptoms on the same data set, but using also self-ratings and peer nominations, Minna Happonen found that estimates of additive genetic effects were significant for both boys and girls across all four types of informants, and these estimates ranged from 0.28 to 0.71. Significant effects from common environmental sources were found only in ratings of teachers and parents, where, typically, one individual rated both co-twins. Sexlimitation effects were found only in teacher ratings, where genetic effects were greater in girls than in boys (Happonen et al., in press).

\section{Future Goals and Designs}

As mentioned above one of our goals is to evaluate and detect gene-environment interactions in behavioral development using a longitudinal twin-family design. The longitudinal aspect of studies with repeated measurements, can be analyzed as if it were a study of individuals, predicting behaviors and health status at a later time from baseline or early follow-up. The data can also be used to evaluate the genetic contribution to interindividual variability of traits, and to the stability or change of a trait over time. The inclusion of parental data permits evaluation of assortative mating effects and generational transmission through the use of twin-family models (Neale \& Cardon, 1992). A challenge for analysis will be combine all aspects of these designs and integrate measured genotypic and specific environmental measures, to provide an integrated picture of the development of the traits of interest with models that are realistic and can take into account the complexity of the data.

The study of the genetics of complex metabolic systems and their disorders is a balance between a reductionist approach, which permits the identification of the components of a system, and on the other hand an integrated approach to the workings of the system as a whole. Studies on complex adaptive systems suggest that while we dissect out the components of systems, this information is not sufficient to form an overview of the entire system (Kauffmann, 1993). That is, the entire system has emergent properties that are not predictable on the basis of knowledge of the components alone: also its future development is conditional on its past history. The linear models used in many genetic and epidemiological studies have often ignored gene-environment interaction as well the dynamical (time-dependent) character of the adaptive system.

A proper analysis of genotype-environment interaction must consider both biological and statistical issues. Genetic differences may determine how an organism responds to changes in the environment. If genotypes respond differently, genotype-environment interactions are inferred (Baker, 1988). These interactions are defined as the failure of the responses of each genotype to different environments to be parallel. The statistical assessment of a biologically meaningful divergence of response will be dependent on sample size. Thus, the biological and statistical criteria for the presence of interaction may not always coincide. A stricter and more conservative criterion for the presence of gene-environment interaction is that the phenotypes associated with the genotypes have different ranks in different environments. Ideally an analysis of genotype-environment interaction would consist of exposing individuals and families of known genotype to different environments and assessing the phenotypic changes in the trait under study, when this is ethically possible. For many environmental exposures and with a long period between exposure and outcome, human studies are limited to observational designs. In these situations, twin and family of twins data can be considered very informative.

\section{Acknowledgments}

We have benefited greatly by contributions from Dorret Boomsma, Peter Molenaar, Richard Viken, Markku Koskenvuo, Jouko Lönnqvist, Urho Kujala, Aila Rissanen, Kalle Romanov and Irma Moilanen.

Support has been given by the Academy of Finland (grants \#27927, 42044, 44069, 100499 ), U.S. - P.H.S (AA-12502, AA-9203, AA 8315, AA-00145), the Yrjö Jahnsson Foundation, the European Union (Factors in Healthy Eating — study: FP5 QLK-1999-916) and the Finnish Foundation for Alcohol Studies. This paper was prepared as a part of the project: "Human Development and Its Risk Factors" financed by the Academy of Finland (Finnish Centre of Excellence Program 2000-2002).

\section{References}

Aarnio, M., Kujala, U. M., \& Kaprio, J. (1997). Associations of health-related behaviors, school type and health status to physical activity patterns in 16 year old boys and girls. Scandinavian Journal of Social Medicine, 25, 156-167.

Aarnio, M., Winter, T., Kujala, U. M., \& Kaprio, J. (1997). Familial aggregation of leisure-time physical activity A three generation study. International Journal of Sports Medicine, 18, 549-556.

Baker, R. J. (1988). Differential response to environmental stress. In B. S.Weir, E. J. Eisen, M. M. Goodman, \& G. Namkoong (Eds.), Proceedings of the second international conference on quantitative genetics (pp. 492-504). Sunderland, Mass.: Sinauer Associates,Inc.

Bucholz, K. K., Cadoret, R., Cloninger, C. R., Dinwiddie, S. H., Hesselbrock, V. M., Nurnberger, J. I., et al. (1994). A new, semi-structured psychiatric interview for use in genetic linkage studies - A report on the reliability of the Ssaga. Journal of Studies on Alcohol, 55, 149-158.

Dick, D. M., Rose, R. J., Viken, R. J., Kaprio, J., \& Koskenvuo, M. (2001). Exploring gene-environment interactions: Socioregional moderation of alcohol use. Journal of Abnormal Psychology, 110, 625-632.

Happonen, M., Pulkkinen, L., Kaprio, J., Van der Meere, J., \& Rose, R. J. (in press). The heritability of depressive symptoms - Multiple informants and multiple measures. Journal of Child Psychology and Psychiatry and Allied Disciplines

Kaprio, J. (1994). Lessons from twin studies in Finland. Annals of Medicine, 26, 135-139.

Kaprio, J., Koskenvuo, M., \& Rose, R. J. (1990). Population-based twin registries: Illustrative applications in genetic epidemiology and behavioral genetics from the Finnish Twin Cohort Study. Acta Geneticae Medicae et Gemellologiae, 39, 427-439. 
Kaprio, J., Rimpelä, A., Winter, T., Viken, R. J., Rimpelä, M., \& Rose, R. J. (1995). Common genetic influences on BMI and age at menarche. Human Biology, 67, 739-753.

Kauffmann, S. A. (1993). The origins of order: Self-organization and selection in evolution. Oxford: Oxford University Press.

Laitinen, T., Räsänen, M., Kaprio, J., Koskenvuo, M., \& Laitinen, L. A. (1998). Importance of genetic factors in adolescent asthma: A population-based twin-family study. American Journal of Respiratory \& Critical Care Medicine, 157, 1073-1078.

Neale, M. C., \& Cardon, L. R. (1992). Methodology for genetic studies of twins and families. Dordrecht: Kluwer Academic.

Pietiläinen, K. H., Kaprio, J., Rissanen, A., Winter, T., Rimpelä, A., Viken, R. J., et al. (1999). Distribution and heritability of BMI in Finnish adolescents aged 16y and 17y: A study of 4884 twins and 2509 singletons. International Journal of Obesity and Related Metabolic Disorders, 23, 107-115.

Pietiläinen, K. H., Kaprio, J., Räsänen, M., Winter, T., Rissanen, A., \& Rose, R. J. (2001). Tracking of body size from birth to late adolescence: contributions of birth length, birth weight, duration of gestation, parents' body size, and twinship. American Journal of Epidemiology, 154, 21-29.

Pulkkinen, L., Kaprio, J., \& Rose, R. J. (1999). Peers, teachers and parents as assessors of the behavioural and emotional problems of twins and their adjustment: The Multidimensional Peer Nomination Inventory. Twin Research, 2, 274-285.

Rose, R. J., Dick, D. M., Viken, R. J., \& Kaprio, J. (2001). Geneenvironment interaction in patterns of adolescent drinking: Regional residency moderates longitudinal influences on alcohol use. Alcoholism, Clinical \& Experimental Research, 25, 637-643.

Rose, R. J., Dick, D. M., Viken, R. J., Pulkkinen, L., \& Kaprio, J. (2001). Drinking or abstaining at age 14? A genetic epidemiological study. Alcoholism, Clinical \& Experimental Research, $25,1594-1604$
Rose, R. J., Kaprio, J., Winter, T., Koskenvuo, M., \& Viken, R. J. (1999). Familial and socioregional environmental effects on abstinence from alcohol at age sixteen. Journal of Studies on Alcohol Supplement, 13, 63-74.

Räsänen, M., Kaprio, J., Laitinen, T., Winter, T., Koskenvuo, M., \& Laitinen, L. A. (2000). Perinatal risk factors for asthma in Finnish adolescent twins. Thorax, 55, 25-31.

Räsänen, M., Laitinen, T., Kaprio, J., Koskenvuo, M., \& Laitinen, L. A. (1997). Hay fever, asthma and number of older siblings - A twin study. Clinical \& Experimental Allergy, 27, 515-518.

Räsänen, M., Laitinen, T., Kaprio, J., Koskenvuo, M., \& Laitinen, L. A. (1998). Hay fever - A Finnish nationwide study of adolescent twins and their parents. Allergy, 53, 885-890.

Seppä, K., Sillanaukee, P., \& Koivula, T. (1990). The efficiency of a questionnaire in detecting heavy drinkers. British Journal of Addiction, 85, 1639-1645.

Silventoinen, K., Kaprio, J., Lahelma, E., Viken, R. J., \& Rose, R. J. (2001). Sex differences in genetic and environmental factors contributing to body-height. Twin Research, 4, 25-29.

Vierikko, E., Pulkkinen, L., Kaprio, J., Rose, R. J., \& Viken, R. J. (in press). Sex differences in genetic and environmental effects on aggression. Aggressive Behaviors.

Winter, T., Kaprio, J., Viken, R. J., Karvonen, S., \& Rose, R. J. (1999). Individual differences in adolescent religiosity in Finland: Familial effects are modified by sex and region of residence. Twin Research, 2, 108-114.

Winter, T., Karvonen, S., \& Rose, R. J. (in press). Does religiousness explain regional differences in alcohol use in Finland? Alcohol and Alcoholism. 